\title{
Statistically Deformable 2D/3D Registration for Accurate Determination of Post-operative Cup Orientation from Single Standard X-ray Radiograph
}

\author{
Guoyan Zheng \\ ISTB, University of Bern, Stauffacherstrasse 78, CH-3014 Bern, Switzerland \\ guoyan.zheng@ieee.org
}

\begin{abstract}
The widely used procedure of evaluation of cup orientation following total hip arthroplasty using single standard anteroposterior (AP) radiograph is known inaccurate, largely due to the wide variability in individual pelvic orientation relative to $\mathrm{X}$-ray plate. $2 \mathrm{D} / 3 \mathrm{D}$ rigid image registration methods have been introduced for an accurate determination of the post-operative cup alignment with respect to an anatomical reference extracted from the CT data. Although encouraging results have been reported, their extensive usage in clinical routine is still limited. This may be explained by their requirement of a CAD model of the prosthesis, which is often difficult to be organized from the manufacturer due to the proprietary issue, and by their requirement of a pre-operative CT scan, which is not available for most retrospective studies. To address these issues, we developed and validated a statistically deformable $2 \mathrm{D} / 3 \mathrm{D}$ registration approach for accurate determination of post-operative cup orientation. No CAD model and pre-operative CT data is required any more. Quantitative and qualitative results evaluated on cadaveric and clinical datasets are given, which indicate the validity of the approach.
\end{abstract}

\section{Introduction}

Two-dimensional (2D) anteroposterior (AP) pelvic radiographs, despite their inferior accuracy in comparison to three-dimensional (3D) techniques based on computed tomography (CT) [1], are the standard imaging method for the evaluation of cup orientation following total hip arthroplasty (THA) [2], largely due to the simplicity, availability, and minimal expense associated with acquiring these images. While plain pelvic radiographs are easily obtained, their accurate interpretation is complicated by the wide variability in individual pelvic orientation relative to the X-ray plate [1]. In THA, increased pelvic tilt results in significant decreases in apparent prosthetic cup anteversion and vice versa [3].

$2 \mathrm{D} / 3 \mathrm{D}$ rigid registration methods [4 5, 6] have been introduced to estimate the post-operative cup alignment with respect to an anatomical reference, which is a plane called the anterior pelvic plane (APP) defined by the anterior superior iliac spines (ASIS) and the pubic tubercles 8. The common procedure within 
these methods is to use $2 \mathrm{D} / 3 \mathrm{D}$ registration to align both the prosthesis and the pre-operative $\mathrm{CT}$ volume to the X-ray image(s), which then allows the position of the implant to be calculated with respect to the anatomical reference plane. Although encouraging results have been reported, their extensive usage in clinical routine is still limited. This may be explained by their requirement of a Computer Aided Design (CAD) model of the prosthesis [4] [5] 6], which often is difficult to be organized from the manufacturer due to the proprietary issue, and by their requirement of a pre-operative CT scan [4] [5] 6], which is not available for most retrospective studies or earlier cup designs. We addressed the former issue in our previous work [7, where a hybrid $2 \mathrm{D} / 3 \mathrm{D}$ registration scheme was implemented to estimate a rigid transformation between a pre-operative CT volume and the post-operative X-ray radiograph. No registration between the prosthesis and the $\mathrm{X}$-ray radiograph is required, thus eliminating the necessity of possessing the CAD models of the prosthesis. In this paper, we would like to address the latter issue through $2 \mathrm{D} / 3 \mathrm{D}$ reconstruction.

Reconstructing a 3D bone model from single $2 \mathrm{D}$ projection image is a challenging task. Furthermore, in our application, no radiograph-specific calibration is available. The only information that we assume to know about the radiograph is the image scale (mm/pixel) and the distance from the focal point to the imaging plane or to the film. As long as the radiograph is acquired in a standardized way, which is preformed in a clinical routine [2, both parameters can be estimated by performing one-time calibration 9 . When only single image is used, it is well-known that the depth information and the scaling factor are correlated with each other. Thus, precise estimation of both of them from single image is difficult, if it is not impossible. However, considering the context of our application, we hypothesize that even a scaled estimation of the pelvic model should provide enough information to determine the post-operative cup orientation. We thus developed a statistically deformable $2 \mathrm{D} / 3 \mathrm{D}$ registration approach to instantiate a patient-specific pelvis surface model from the single standard $\mathrm{X}$-ray radiograph and then used the instantiated surface model to determine the post-operative cup orientation.

This paper is organized as follows. Section 2 briefly presents the construction of the statistical shape model. Section 3 describes the statistically deformable $2 \mathrm{D} / 3 \mathrm{D}$ registration approach. Section 4 presents the experimental results, followed by the conclusions in Section 5 .

\section{Construction of the Statistical Shape Model of the Pelvis}

We chose the point distribution model (PDM) as the representation of our statistical shape model of the pelvis. The pelvic PDM used in this paper was constructed from a training database consisted of 14 segmented binary volumes (12 of them were segmented from CT scans of dry bones and the rest 2 were segmented from patient CT scans) where the sacrum was removed from each dataset. Demon's algorithm [10] as implemented in MedINRIA [11] was used to 

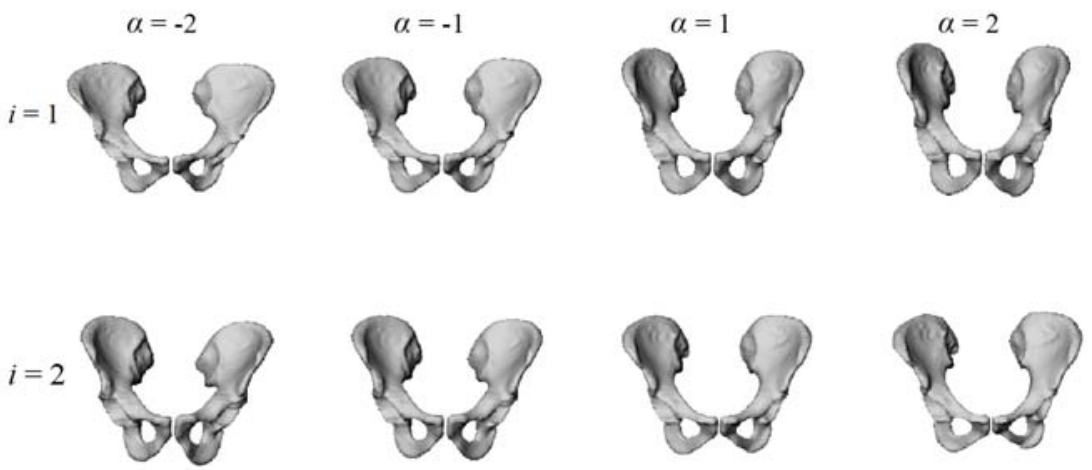

Fig. 1. The first two eigen modes of variation of our PDM of the pelvis. The shape instances were generated by evaluating $\bar{x}+\alpha \sigma_{i} \mathbf{p}_{i}$ with $\alpha \in\{-2,-1,1,2\}$.

estimate the dense deformation fields between the reference binary volume and the other 13 binary volumes. Each estimated deformation field was then used to displace the positions of the vertices on the reference surface model, which was constructed from the reference binary volume, to the associated target volume. We thus obtained 14 surface models with established correspondences.

Following the alignment, the PDM is constructed as follows. Let $\mathrm{x}_{i}, i=$ $0,1, \ldots, m-1$, be $m$ (here $m=14$ ) members of the aligned training surfaces. Each member is described by a vectors $\mathrm{x}_{i}$ with $N$ (here $N=24994$ ) vertices:

$$
\mathrm{x}_{i}=\left\{x_{0}, y_{0}, z_{0}, x_{1}, y_{1}, z_{1}, \ldots, x_{N-1}, y_{N-1}, z_{N-1}\right\}
$$

The PDM is obtained by applying principal component analysis.

$$
\begin{aligned}
& D=\left((m-1)^{-1}\right) \cdot \sum_{i=0}^{m-1}\left(\mathrm{x}_{i}-\overline{\mathrm{x}}\right)\left(\mathrm{x}_{i}-\overline{\mathrm{x}}\right)^{T} \\
& P=\left(\mathbf{p}_{0}, \mathbf{p}_{1}, \ldots\right) ; D \cdot \mathbf{p}_{i}=\sigma_{i}^{2} \cdot \mathbf{p}_{i}
\end{aligned}
$$

where $\overline{\mathrm{x}}$ and $D$ are the mean vector and the covariance matrix, respectively.

Fig 1 shows the variability captured by the first two modes of variation of our PDM.

\section{Statistically Deformable 2D/3D Registration}

Our single image based surface model reconstruction technique is based on the algorithm that we introduced in [12, which combines statistical instantiation and regularized shape deformation with an iterative image-to-model correspondence establishing algorithm. The image-to-model correspondence is established using a non-rigid $2 \mathrm{D}$ point matching process, which iteratively uses a symmetric injective nearest-neighbor mapping operator and $2 \mathrm{D}$ thin-plate splines based deformation to find a fraction of best matched 2D point pairs between features extracted from the X-ray images and the projections of the apparent contours 


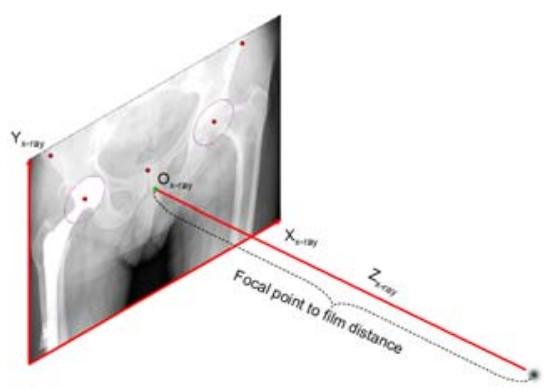

(a)

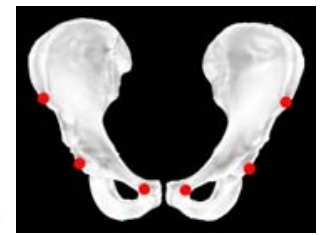

(b)

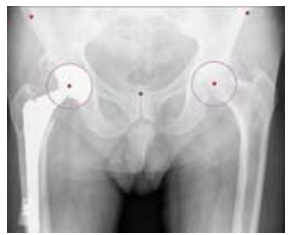

(c)

Fig. 2. (a) The radiograph coordinate system and the cone-beam projection model; (b) Landmarks extracted from the mean model of the PDM; and (c) landmarks extracted from radiograph

extracted from the 3D model. The obtained 2D point pairs are then used to set up a set of $3 \mathrm{D}$ point pairs such that we turn a $2 \mathrm{D} / 3 \mathrm{D}$ reconstruction problem to a $3 \mathrm{D}-3 \mathrm{D}$ one. The $3 \mathrm{D} / 3 \mathrm{D}$ reconstruction problem is then solved optimally in three sequential stages including iterative scaled rigid registration, statistical instantiation, and regularized shape deformation. For details, we refer to our previous works [12].

In our previous work, we asked for 2 or more X-ray images as the input and that all images should be calibrated. However, these requirements are the conditions for the application in our previous work rather than the constraints to our algorithm. Actually, the algorithm that we introduced in [12] can be directly applied to single image, as long as at least four non-colinear point pairs are found. Due to the fact that only single image is available, we may not obtain precise reconstructions as those reported in our previous work, but rather scaled reconstructions. Similar to the situation when multiple images are used, the convergence of the single image based $2 \mathrm{D} / 3 \mathrm{D}$ reconstruction also depends on the initialization. Thus, in the following we focus on the establishment of the projection geometry of the input radiograph, and on a landmark-based scaled registration for initializing the single image based $2 \mathrm{D} / 3 \mathrm{D}$ reconstruction.

\subsection{Establishment of Projection Geometry}

The local coordinate reference and the cone-beam projection model of the radiograph is established as follows (see Fig. 2(a) for details). The image center is taken as the coordinate origin. The $\mathrm{X}$-axis and the $\mathrm{Y}$-axis of the image are taken as the $\mathrm{X}$-axis and the $\mathrm{Y}$-axis of the local coordinate reference of the radiograph. The central projection line is perpendicular to the radiograph plane and its opposite direction is regarded as Z-axis.

\subsection{Landmark-Based Scaled Registration for Initialization}

Initialization here means to estimate the initial scale and the rigid transformation between the mean model of the PDM and the input radiograph. For 
this purpose, we have adopted an iterative landmark-to-ray scaled registration. The five anatomical landmarks that we used here are left and right ASIS, left and right acetabular centers, and pubic symphysis. Their positions on the mean model of the PDM are obtained through point picking (for left and right ASIS, and pubic symphysis) or sphere fitting (for left and right acetabular centers), while their positions on the radiograph are defined through interactive picking (for the projections of left and right ASIS, and pubic symphysis) or circle fitting (for the projections of left and right acetabular centers) (see Fig. 2(b) and 2(c) for details).

Let us denote those landmarks defined on the mean model of the PDM, i.e., the left and the right acetabular centers, the pubic symphysis, and the middle points of the left and the right ASIS, as $v_{\text {Mean }}^{1}, v_{\text {Mean }}^{2}, v_{\text {Mean }}^{3}$, and $v_{\text {Mean }}^{4}$, respectively; and their corresponding landmarks interactively picked from the radiograph as $v_{X-r a y}^{1}, v_{X-r a y}^{2}, v_{X-\text { ray }}^{3}$, and $v_{X-\text { ray }}^{4}\left(v_{X-\text { ray }}^{4}\right.$ is the middle point of the projections of the left and the right ASIS), respectively. And for each $\mathrm{X}$-ray landmark, we can calculate a projection ray emitting from the focal point to the landmark. We then calculate the length between $v_{M e a n}^{1}$ and $v_{M e a n}^{2}$ and denote it as $l_{\text {Mean }}^{1,2}$. Using the known image scale, we also calculate the length $l_{X-\text { ray }}^{1,2}$ between $v_{X-\text { ray }}^{1}$ and $v_{X-\text { ray }}^{2}$. Then, we do:

Data Preparation. In this step, we assume that the line connecting the acetabular centers is parallel to the AP pelvic radiograph plane and is certain distance away from the imaging plane or the film (in all the experiments reported in this paper, we used a fixed distance of $150 \mathrm{~mm}$ ). Using this assumption and the correspondences between the landmarks defined in the CT volume and those picked from the radiograph, we can first compute two points $\bar{v}_{X-\text { ray }}^{1}$ and $\bar{v}_{X-\text { ray }}^{2}$ on the projection rays of $v_{X-\text { ray }}^{1}$ and $v_{X-\text { ray }}^{2}$, respectively, which satisfy:

$$
\bar{v}_{X-\text { ray }}^{1} \bar{v}_{X-\text { ray }}^{2} / / v_{X-r a y}^{1} v_{X-r a y}^{2} ; \text { and }\left|\bar{v}_{X-\text { ray }}^{1}-\bar{v}_{X-\text { ray }}^{2}\right|=l_{X-r a y}^{1,2} \times \frac{F-d}{F}
$$

where $F$ is the known distance from the focal point to the imaging plane and $d$ is the assuming distance from the acetabular centers to the imaging plane.

The current scale $s$ between the mean model and the input image is then estimated as,

$$
s=\left|\bar{v}_{X-\text { ray }}^{1}-\bar{v}_{X-\text { ray }}^{2}\right| / l_{\text {Mean }}^{1,2}
$$

Using $s$, we scale all landmark positions on the mean model and denote them as $\left\{\bar{v}_{\text {Mean }}^{i} ; \mathrm{i}=1,2,3,4\right\}$. We then calculate the distances from $\bar{v}_{\text {Mean }}^{3}$ and $\bar{v}_{\text {Mean }}^{4}$ to line $\bar{v}_{\text {Mean }}^{1} \bar{v}_{\text {Mean }}^{2}$ and denote it as $\bar{l}_{\text {Mean }}^{3,1-2}$ and $\bar{l}_{\text {Mean }}^{4,1-2}$, respectively.

Next we find two points, point $\bar{v}_{X-\text { ray }}^{3}$ on the projection ray of $v_{X-\text { ray }}^{3}$ whose distance to the line $\bar{v}_{X-\text { ray }}^{1} \bar{v}_{X-\text { ray }}^{2}$ is equal to $\vec{l}_{\text {Mean }}^{3,1-2}$, and point $\bar{v}_{X-\text { ray }}^{4}$ on the projection ray of $v_{X-\text { ray }}^{4}$ whose distance to the line $\bar{v}_{X-\text { ray }}^{1} \bar{v}_{X-\text { ray }}^{2}$ is equal to $\bar{l}_{\text {Mean }}^{4,1-2}$. A paired-point matching [13] based on $\left\{\bar{v}_{\text {Mean }}^{i} ; \mathrm{i}=1,2,3,4\right\}$ and $\left\{\bar{v}_{X-\text { ray }}^{i}\right.$; $\mathrm{i}=1,2,3,4\}$ is used to calculate a updated scale $s_{0}$ and a rigid transformation $\bar{T}_{\text {Mean }}^{X-\text { ray }}$ (see Fig. 3(a) for details). From now on, we assume that all information defined in the mean model coordinate frame has been transformed into the 




(a)

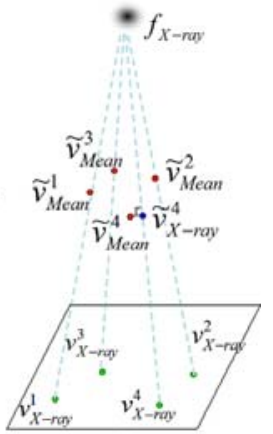

(b)

Fig. 3. Iterative landmark-to-ray registration. (a) schematic view of data preparation; and $(\mathrm{b})$ schematic view of finding $3 \mathrm{D}$ point pairs.

radiograph coordinate frame using $s_{0}$ and $\bar{T}_{M e a n}^{X-\text { ray }}$. We denote the transformed mean model landmarks as $\left\{\tilde{v}_{\text {Mean }}^{i}\right\}$.

Iteration. The following steps are iteratively executed until convergence:

1. For a point $\tilde{v}_{M e a n}^{i}$, we find a point on the corresponding projection ray of $v_{X-\text { ray }}^{i}$ which has the shortest distance to the point $\tilde{v}_{\text {Mean }}^{i}$ and denote it as $\tilde{v}_{X-\text { ray }}^{i}$ (see Fig. 3(b)). We then perform a paired-point matching [13] using the extracted point pairs to compute a scale $\tilde{s}$ and a rigid transformation $\Delta \tilde{T}_{\text {Mean }}^{X-\text { ray }}$.

2. We update the mean model coordinate frame using $\tilde{s}$ and $\Delta \tilde{T}_{\text {Mean }}^{X-\text { ray }}$.

\section{$3.32 \mathrm{D} / 3 \mathrm{D}$ Reconstruction}

The estimated scale and the rigid transformation between the mean model and the input image are then treated as the starting values for the algorithm that we introduced in [12. As a feature-based 2D/3D reconstruction approach, our algorithm requires a pre-requisite image feature extraction. In this paper, observing the superimposition of the projections of different bone structures around the pelvis and the post-operative characteristic of the X-ray radiograph, we opt for an interactive way to identify contours of the pelvis. We thus developed a program allowing the user to define up to eight contours by interactively picking points from the radiograph. Each contour is then interpolated by a cubic-spline to have the same resolution as the image resolution. The extracted contours are then used together with the initial estimation of the scale and the rigid transformation as the input to our PDM based 2D/3D reconstruction scheme for an accurate reconstruction of a surface model of the pelvis. Fig. 4 shows different stages of reconstruction of a patient-specific surface model of the pelvis from single standard X-ray radiograph of a patient. The reconstructed surface model of the pelvis can then be used to determine the post-operative cup orientation. 


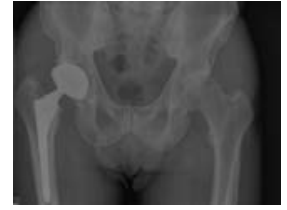

(a)

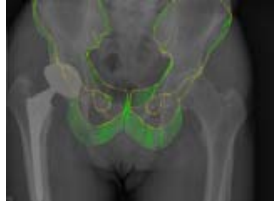

(b)



(c)

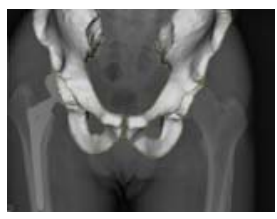

(d)

Fig. 4. (a) the image contours (white line); (b) establishment of the initial image-tomodel correspondences (yellow points: projections of the apparent contours extracted from the mean model; green lines: visualization of the correspondences); (c) the result of the iterative scaled registration; and $(d)$ the final reconstructed surface model

Table 1. Difference between the estimated results estimated and the ground truths

\begin{tabular}{l|c|c|c|c|c|c|c}
\hline \hline angle & cadaver_01 & cadaver_02 & cadaver_03 & cadaver_04 & Patient_01 & Patient_02 & Mean \\
\hline anteversion $\left({ }^{\circ}\right)$ & 1.0 & 4.4 & 3.0 & 1.0 & 1.5 & 1.2 & $2.0 \pm 1.4$ \\
\hline inclincation $\left({ }^{\circ}\right)$ & 0.9 & 1.1 & 0.8 & 0.7 & 0.8 & 2.0 & $1.4 \pm 0.5$ \\
\hline
\end{tabular}

\section{Experiments and Results}

We designed and conducted experiments on 4 cadaveric pelvis datasets and 2 patient datasets (one male and one female. Note: none of them has been included for constructing the PDM) to validate the present approach. Each dataset contains a post-operative X-ray radiograph and a post-operative CT volume. The cup orientations extracted from the associated post-operative CT volume are regarded as the ground truths. The results obtained by the present approach are compared to the associated ground truths to estimate the measurement errors. Two X-ray machines were used to acquire the X-ray radiograph. The X-ray radiographs for all 4 cadaveric pelvis were acquired by one X-ray machine with a focal point to film distance of $1200 \mathrm{~mm}$ and a pixel size of $0.143 \mathrm{~mm}$ while the X-ray radiographs for two patients were acquired by the other X-ray machine with a focal point to film distance of $1016 \mathrm{~mm}$ and a pixel size of $0.17 \mathrm{~mm}$.

The results of our validation experiment are presented in Table 1 . Compared to the ground truths, differences of $2.0^{\circ} \pm 1.4^{\circ}$ were found for the anteversion and differences of $1.4^{\circ} \pm 0.5^{\circ}$ were found for the inclination. Although the anteversion measurement errors are slightly bigger than those based on pre-operative $\mathrm{CT}$ scans [7, they are still in the acceptable range according to Kalteis et al. [1].

\section{Conclusions}

In this paper, we presented a statistically deformable $2 \mathrm{D} / 3 \mathrm{D}$ registration approach to instantiate a patient-specific pelvis surface model from single standard $\mathrm{X}$-ray radiograph and then used the reconstructed model to determine the postoperative cup orientation. We designed and conducted feasibility experiments to validate the present approach. Our experimental results demonstrate that it 
is feasible to reconstruct a patient-specific model from single standard X-ray radiograph for accurate determination of post-operative cup orientation.

\section{References}

1. Kalteis, T., Handel, M., Herold, T., Perlick, L., Paetzel, C., Grifka, J.: Position of the acetabular cup-accuracy of radiographic calculation compared to CT-based measurement. Eur. J. Radiol. 58, 294-300 (2006)

2. Della Valle, C.J., Kaplan, K., Jazrawi, A., Ahmed, S., Jaffe, W.L.: Primary total hip arthroplasty with a flanged, cemented all-polyethylene acetabular component. J. Arthroplasty 19, 23-26 (2004)

3. Sellers, R.G., Lyles, D., Dorr, L.D.: The effect of pelvic rotation on alpha and theta angles in total hip arthroplasty. Contemp. Orthop. 17, 67-70 (1988)

4. Blendea, S., Eckman, K., Jaramaz, B., Levison, T.J., DiGioia, A.M.: Measurements of acetabular cup position and pelvic spatial orientation after total hip arthroplasty using computed tomography/radiography matching. Computer Aided Surgery 10, $37-43(2005)$

5. Jaramaz, B., Eckman, K.: 2D/3D registration for measurement of implant alignment after total hip replacement. In: Larsen, R., Nielsen, M., Sporring, J. (eds.) MICCAI 2006. LNCS, vol. 4191, pp. 653-661. Springer, Heidelberg (2006)

6. Penney, G.P., Edwards, P.J., Hipwell, J.H., Slomczykowski, M., Revie, I., Hawkes, D.J.: Postoperative calculation of acetabular cup position using 2D - 3D registration. IEEE T. Biomed. Eng. 54, 1342-1348 (2007)

7. Zheng, G., Steppacher, S., Zhang, X., Tannast, M.: Precise estimation of postoperative cup alignment from single standard X-ray radiograph. In: Ayache, N., Ourselin, S., Maeder, A. (eds.) MICCAI 2007, Part II. LNCS, vol. 4792, pp. 951959. Springer, Heidelberg (2007)

8. Jaramaz, B., DiGioia, A.M., Blackwell, M., Nikou, C.: Computer assisted measurement of cup placement in total hip replacement. Clin. Orthop. Rel. Res. 354, 70-81 (1998)

9. The, B.: Digital radiographic peroperative planning and postoperative monitoring of total hip replacements. Doctoral dissertations, University Medical Center Groningen, the Netherlands (2006)

10. Thirion, J.-P.: Image matching as a diffusion process: an analogy with Maxwell's demons. Med. Image Anal. 2, 243-260 (1998)

11. Toussaint, N., Souplet, J.-C., Fillard, P.: MedINRIA: DT-MRI processing and visualization software. In: MICCAI 2007 Workshop on Interaction in Medical Image and Visualization (2007)

12. Zheng, G., Ballester, M.A.G., Styner, M.A., Nolte, L.-P.: Reconstruction of patientspecific $3 \mathrm{D}$ bone surface from $2 \mathrm{D}$ calibrated fluoroscopic images and point distribution model. In: Larsen, R., Nielsen, M., Sporring, J. (eds.) MICCAI 2006. LNCS, vol. 4190, pp. 25-32. Springer, Heidelberg (2006)

13. Veldpaus, F.E., Woltring, H.J., Dortmans, L.J.: A least-square algorithm for the equiform transformation from spatial marker coordinates. J. Biomech. 21, 45-54 (1988) 